\title{
COMPARATIVE ANALYSIS OF ROUTING PROTOCOLS AND IP TRAFFIC MANAGEMENT USING ACL
}

\author{
Shreehitha M U \\ Dept. of Electronics and Communication \\ RV College of Engineering, Bengaluru, Karnataka, \\ India
}

\begin{abstract}
The aim of the study is to compare the interior routing protocols RIP, OSPF \& EIGRP considering network parameters like metrics, Convergence time \& scalability. All the three protocols are configured for a common hybrid network. The study make use of standard and extended ACLs.
\end{abstract}

Keywords-RIP, OSPF, EIGRP, ACL, Cisco Packet Tracer

\section{INTRODUCTION}

Routing protocols should be very well planned and dimensioned for each type of network. They are used according to the choices of the network administrator. The dynamic routing protocols are divided in two distinct categories: interior routing protocols and exterior routing protocols. Interior routing protocols are classified as distance vector and link-state protocols. RIPv1, RIPv2, and EIGRP belong to Distance vector protocol. Cisco provides Access Control Lists (ACLs) to control the flow of traffic from one interface to the other in the network. ACL also performs other tasks such as restricting telnet, filtering routing information and prioritizing WAN traffic with queuing. A wildcard mask allows to match the range of address in the ACL statements. A router makes two references to ACL such as numbered and named. These references support two types of filtering such as standard and extended. The ACL statements are configured first and then they are activated. The benefits of ACL are reduced network traffic and increase network performance and control the flow of traffic. A simple network topology is simulated with all three routing protocols and collect statistics such as convergence time and routing traffic sent. The routing tables of a simple network topology is studied to understand the metrics of each protocol and gain a better insight on how routes are chosen.

C.G. Dumitrache, G. Predusca [1] Considering the executed simulations and the gained outcomes the paper conclude that the most efficient protocol is EIGRP because uses a less complicated algorithm than the one OSPF does; this one is very well scaled on the middle-sized networks and well on the big-sized networks, while OSPF is very well

\author{
Dr. Kiran V \\ Associate Professor \\ Dept. of Electronics and Communication \\ RV College of Engineering, Bengaluru, Karnataka \\ India
}

scaled both on the middle-sized and big-sized networks, the latter computing the shortest route.

Nurhayati; Rahmat Fajar Al Farizky [2] Based on the performance evaluation results that have been done by the author on the routing protocol RIPng, OSPFv3, and EIGRP for IPv6 for streaming video services, a number of conclusions were made such as the lower the value of screen resolution (pixels) and bit rate (kbps), the greater the value of throughput (kbps) generated. Value throughput affects the delay and packet loss, throughput of greater value to produce a value of delay and packet loss smaller.

Kavya Altefi; Amir Hossein Shahin [3] this paper presented the modelling of the two EIGRP and RIP routing protocols throughout a802.3u network had been established based on important metric such as throughput, delay and utilization.

Shipra suman aditi Agarwal [4] This paper shows the configuration of standard ACL and extended ACL on the router. The standard ACL create filters based on source addresses only and are used for server-based filtering, whereas extended ACL provide more security by creating filters based on source addresses as well as destination addresses, protocol and port number. The extended ACL in this paper used TCP/IP protocol. Routing Information Protocol (RIP) is used for routing the packets.

Lalu Zazuli Azhar Mardedi Abidarin Rosidi [5] In this paper the results of testing Ring network topology on EIGRP and OSPF routing protocol had the following conclusions. In a ring topology testing the score of total delay EIGRP scores better than OSPF. OSPF is a link-state so that at the same cost value of the package to be delivered is not always the shortest route but also the longest route.

C-C Chiang et al. [6] here the author talks about the security issues, the security mechanisms play an important role in networks and in the Internet world. There are many ways to find vulnerabilities in a network and launch attacks against the network. In this thesis, the authors examine the performance and security problems of several existing routing protocols including RIP, OSPF and EIGRP. Several routing 
performance parameters are evaluated and analysed through using SNMP (Simple Network Management Protocol) sessions. They briefly describe the three IGP protocols, their network Infrastructure and the experimental evaluation methods. In opposite of denegation of service (DOS) attacks and contaminated tables, which are among the most serious attacks to network topologies, the authors propose an automatic mechanism to analyze the states of routing and intrusion detection in real-time response. The study concludes that the distance vector routing protocols are more robust than link-state routing protocols for the unstable network topology because global link-state's flooding of updates increase when link state changes. But the distance vector algorithms can only be used for small networks.

\section{OVER VIEW OF THE ROUTING PROTOCOLS AND ACL}

In IP networks, the main task of a routing protocol is to carry packets forwarded from one node to another. In a network, routing can be defined as transmitting information from a source to a destination by hopping one-hop or multi hop. Conventional routing protocols used in interior gateway networks are classified as Link State Routing Protocols and Distance Vector Routing Protocols. Dynamic routing protocols are of two types Exterior gateway protocol and Interior gateway protocol. Interior gateway protocol are further divided into Distance Vector protocol (e.g. RIP, EIGRP) and Link state protocols (e.g. OSPF).

\section{A. Routing Information Protocol}

The Routing Information Protocol (RIP), which is a distance-vector based algorithm, is one of the first routing protocols implemented on TCP/IP. Information is sent through the network using UDP. Each router that uses this protocol has limited knowledge of the network around it. This simple protocol uses a hop count mechanism to find an optimal path for packet routing. A maximum number of 16 hops are employed to avoid routing loops. However, this parameter limits the size of the networks that this protocol can support. The popularity of this protocol is largely due to its simplicity and its easy configurability. However, its disadvantages include slow convergence times, and its scalability limitations. Therefore, this protocol works best for small-scaled networks.

\section{B. Enhanced Interior Gateway Routing Protocol (EIGRP)}

EIGRP is a Cisco-developed advanced distance-vector routing protocol. Routers using this protocol automatically distribute route information to all neighbours. The Diffusing Update Algorithm (DUA) is used for routing optimization, fast convergence, as well as to avoid routing loops. Full routing information is only exchanged once upon neighbour establishment, after which only partial updates are sent. When a router is unable to find a path through the network, it sends out a query to its neighbours, which propagates until a suitable route is found. This need-based update is an advantage over other protocols as it reduces traffic between routers and therefore saves bandwidth. The metric that is used to find an optimal path is calculated with variables bandwidth, load, delay and reliability. By incorporating many such variables, the protocol ensures that the best path is found. Also, compared to other distance-vector algorithms, EIGRP has a larger maximum hop limitation, which makes it compatible with large networks. The disadvantage of EIGRP is that it is a Cisco proprietary protocol, meaning it is only compatible with Cisco technology.

\section{Open Shortest Path First (OSPF)}

Open Shortest Path First (OSPF) is a very widely used link-state interior gateway protocols (IGP). This protocol routes Internet Protocol (IP) packets by gathering link-state information from neighbouring routers and constructing a map of the network. OSPF routers send many message types including hello messages, link state requests and updates and database descriptions. Dijkstra's algorithm is then used to find the shortest path to the destination. Shortest Path First (SPF) calculations are computed either periodically or upon a received Link State Advertisement (LSA), depending on the protocol implementation. Topology changes are Dynamic Routing Protocols EGP BGP IGP Distance Vector RIP EIGRP Link--State OSPF. Another advantage of OSPF is that its many configurable parameters make it a very flexible and robust protocol. Contrary to RIP, however, OSPF has the disadvantage of being too complicated.

\section{Access-List ACL}

Access-list (ACL) is a set of rules defined for controlling the network traffic and reducing network attack. ACLs are used to filter traffic based on the set of rules defined for the incoming or outgoing of the network. Standard Access-list These are the Access-list which are made using the source IP address only. These ACLs permit or deny the entire protocol suite. They don't distinguish between the IP traffic such as TCP, UDP and Https etc. By using numbers 1-99 or 13001999, router will understand it as a standard ACL and the specified address as source IP address. Extended Access-list These are the ACL which uses both source and destination IP address. In this type of ACL, we can also mention which IP traffic should be allowed or denied. These use range 100-199 and 2000-2699.

\section{Methodology OF ROUTING PROTOCOLS AND ACL}

The mentioned implementations are implemented using Cisco Packet Tracer. The routing protocols are configured for hybrid network topology and comparison is done based on parameters like metrics, convergence time. The access control lists of two types are implemented for 2 scenarios to manage the IP traffic in the network.

\section{A. Implementation of RIP protocol}

RIP v2 is implemented using Cisco packet tracer. Network with hybrid topology is configured as per the Addressing table and RIP v2 protocol is configured for each router. The 
network topology and addressing table is as shown in Fig. 1 and table. 1

\section{$B$. Implementation of OSPF protocol}

OSPF protocol is implemented for a hybrid network topology using Cisco packet tracer. Network is configured based on the address table and OSPF protocol is configured in each of the routers. OSPF for a single area is configured. The Network topology and addressing table is as shown Table. 2

\section{Implementation of EIGRP Protocol}

EIGRP protocol is implemented for a hybrid network topology using Cisco packet tracer. Network is configured based on the address table and EIGRP protocol is configured in each of the routers as similar as RIP and OSPF.

TABLE I. ADDRESSING TABLE FOR RIP PROTOCOL

\begin{tabular}{|c|c|c|c|}
\hline Device & Interfae & $\begin{array}{c}\text { IP } \\
\text { Address }\end{array}$ & Subnet Mask \\
\hline $\mathrm{PC} 0$ & $\mathrm{Fa} 0 / 0$ & 192.168 .1 .10 & 255.255 .255 .0 \\
\hline $\mathrm{PC} 1$ & $\mathrm{Fa} 0 / 0$ & 192.168 .2 .10 & 255.255 .255 .0 \\
\hline $\mathrm{PC} 2$ & $\mathrm{Fa} 0 / 0$ & 192.168 .3 .10 & 255.255 .255 .0 \\
\hline $\begin{array}{c}\text { Router } \\
0\end{array}$ & $\mathrm{Fa} 0 / 0$ & 192.168 .1 .1 & 255.255 .255 .0 \\
\cline { 2 - 4 } & $\mathrm{Se} 2 / 0$ & 172.16 .1 .1 & 255.255 .255 .252 \\
\cline { 2 - 4 } & $\mathrm{Se} 3 / 0$ & 172.16 .1 .10 & 255.255 .255 .252 \\
\hline \multirow{2}{*}{\begin{tabular}{c} 
Router \\
\cline { 2 - 4 }
\end{tabular}} & $\mathrm{Fa} 0 / 0$ & 192.168 .2 .1 & 255.255 .255 .0 \\
\cline { 2 - 4 } & $\mathrm{Se} 2 / 0$ & 172.16 .1 .2 & 255.255 .255 .252 \\
\hline \multirow{2}{*}{$\begin{array}{c}\text { Router } \\
2\end{array}$} & $\mathrm{Fa} 0 / 0$ & 172.16 .1 .5 & 255.255 .255 .252 \\
\cline { 2 - 4 } & $\mathrm{Se} 2 / 0$ & 172.16 .1 .6 & 255.255 .255 .252 \\
\cline { 2 - 4 } & $\mathrm{Se} 3 / 0$ & 172.16 .1 .9 & 255.255 .255 .252 \\
\hline
\end{tabular}

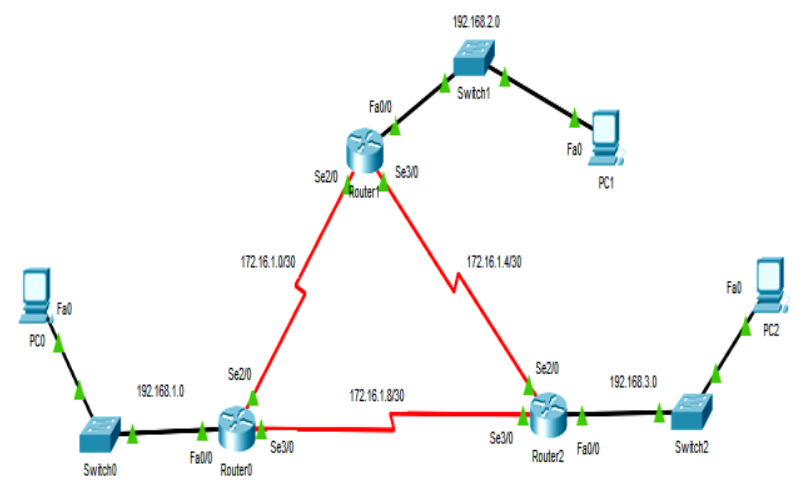

Fig. 1. Network Topology for RIP/OSPF/EIGRP configuration
TABLE II. ADDRESSING TABLE FOR OSPF CONFIGURATION

\begin{tabular}{|l|l|l|l|}
\hline Device & Interface & IP Address & Subnet Mask \\
\hline $\mathrm{PC} 0$ & $\mathrm{Fa} 0 / 0$ & 192.168 .1 .10 & 255.255 .255 .0 \\
\hline $\mathrm{PC} 1$ & $\mathrm{Fa} 0 / 0$ & 192.168 .2 .10 & 255.255 .255 .0 \\
\hline $\mathrm{PC} 2$ & $\mathrm{Fa} 0 / 0$ & 192.168 .3 .10 & 255.255 .255 .0 \\
\hline $\begin{array}{l}\text { Router } \\
0\end{array}$ & $\mathrm{Fa} 0 / 0$ & 192.168 .1 .1 & 255.255 .255 .0 \\
\cline { 2 - 4 } & $\mathrm{Se} 2 / 0$ & 172.16 .1 .1 & 255.255 .255 .252 \\
\cline { 2 - 4 } & $\mathrm{Se} 3 / 0$ & 172.16 .1 .10 & 255.255 .255 .252 \\
\hline \multirow{3}{*}{\begin{tabular}{l} 
Router \\
\cline { 2 - 4 }
\end{tabular}} & $\mathrm{Fa} 0 / 0$ & 192.168 .2 .1 & 255.255 .255 .0 \\
\cline { 2 - 4 } & $\mathrm{Se} 2 / 0$ & 172.16 .1 .2 & 255.255 .255 .252 \\
\cline { 2 - 4 } & $\mathrm{Se} 3 / 0$ & 172.16 .1 .5 & 255.255 .255 .252 \\
\hline \multirow{2}{*}{\begin{tabular}{l} 
Router \\
\cline { 2 - 4 }
\end{tabular}} & $\mathrm{Fa} 0 / 0$ & 192.168 .3 .1 & 255.255 .255 .0 \\
\cline { 2 - 4 } & $\mathrm{Se} 2 / 0$ & 172.16 .1 .6 & 255.255 .255 .252 \\
\cline { 2 - 4 } & $\mathrm{Se} 3 / 0$ & 172.16 .1 .9 & 255.255 .255 .252 \\
\hline
\end{tabular}

IV. IMPLEMENTATION OF IP TRAFFIC MANAGEMENT There are two techniques for managing IP traffic using ACL.

\section{A. Implementation of IP traffic management using standard ACL}

Standard access control lists (ACLs) are router configuration scripts that control whether a router permits or denies packets based on the source address. Standard ACL is

implemented using Cisco packet tracer in order to control the network of traffic by allowing or denying traffic coming from specific network or hosts.

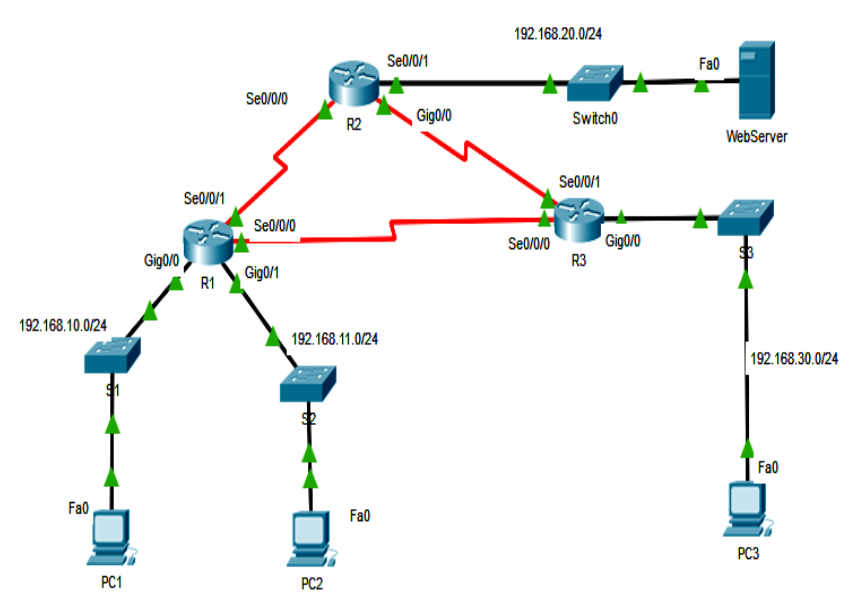

Fig. 2 Network Topology for Standard ACL 


\section{$B$. Implementation of IP traffic management using} Extended ACL

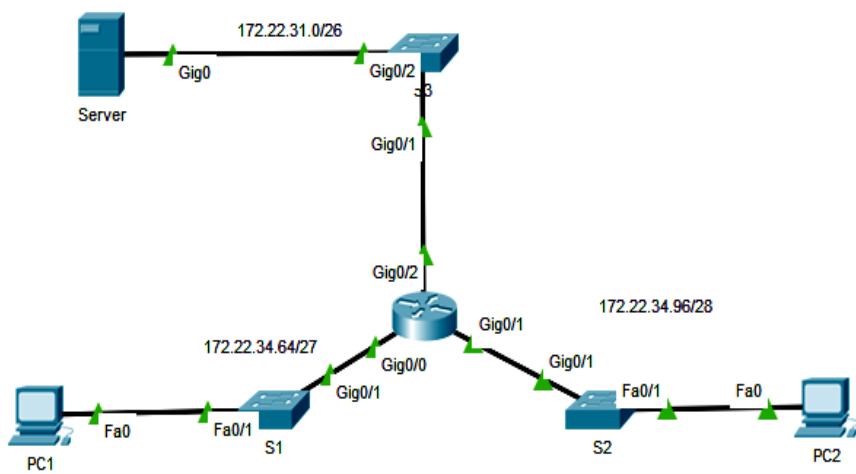

Fig. 3 Network Topology for Extended ACL

- Verify the RIP, OSPF, EIGRP and ACL implementation.

\section{RESULTS}

First the routing protocols RIP, OSPF \& EIGRP are compared based on the parameters like metrics(cost), convergence time \& the best suited protocol is obtained. Next an IP network's traffic is controlled using standard ACL and extended ACL.

\section{A. Comparison of rouing protocols using network Parameters}

RIP version 2: Network parameters that are used for comparison are Metrics, Convergence time \& Scalability. RIP uses hop count as the metric (that defines the cost to reach to destination). RIP has a maximum hop count of 15 hops. Any network that is 16 hops away or more is considered unreachable to RIP, thus the maximum diameter of the network is 15 hops.

Convergence time is the time which a group of routers reach the state of convergence. Optimally the routing protocols must have fast convergence time. RIP v2 is said to have lower convergence time.

From the above observation, for the network topology configured RIP v2 takes $17.658 \mathrm{sec}$ to converge.

Scalability is another network parameter that judges the performance parameter of a routing protocol. In RIP v2 the maximum hop count i.e., the maximum number of routers that can be considered inside a network is 15 . Hence RIP v2 is only suitable for small networks and cannot be applied to large networks.

OSPF Protocol: OSPF protocol uses cost as metric, Cisco cost for OSPF is given by the cost equation: Cisco IOS Cost for SPF $=10^{\wedge} 8 /$ bandwidth in bps The Cost of each interface (branch) is assigned using Ip cost command or by assigning different bandwidth value for each interface. In the above case Router0 has 2 interfaces se $2 / 0$ with cost 64 and se $3 / 0$ with cost 1562. Router 1 has interfaces se $2 / 0$ with cost 64 and se $3 / 0$ with cost 64 . Router 2 has interface of se $2 / 0$ with cost 64 . The next parameter considered for comparison is convergence time. Convergence time of OSPF protocol configured network is as shown in Fig. 5

\begin{tabular}{|c|c|c|c|c|}
\hline \multicolumn{5}{|c|}{ Event List } \\
\hline \multirow[t]{21}{*}{\begin{tabular}{|l} 
Vis. \\
\end{tabular}} & Time(sec) & Last Device & At Device & Type \\
\hline & 8.713 & - & Router2 & RIPv2 \\
\hline & 8.713 & - & Router2 & RIPv2 \\
\hline & 8.713 & - & Router2 & RIPv2 \\
\hline & 8.714 & Router2 & Router1 & RIPv2 \\
\hline & 8.714 & Router2 & Routero & RIPv2 \\
\hline & 8.714 & Router2 & Switch2 & RIPv2 \\
\hline & 8.715 & Switch2 & $\mathrm{PC} 2$ & RIPv2 \\
\hline & 15.715 & - & Router1 & RIPv2 \\
\hline & 15.715 & - & Router1 & RIPv2 \\
\hline & 15.715 & - & Router1 & RIPv2 \\
\hline & 15.716 & Router1 & Switch1 & RIPv2 \\
\hline & 15.716 & Router1 & Routero & RIPv2 \\
\hline & 15.716 & Router1 & Router2 & RIPv2 \\
\hline & 15.717 & Switch1 & PC1 & RIPv2 \\
\hline & 17.656 & - & Routero & RIPv2 \\
\hline & 17.656 & -- & Routero & RIPv2 \\
\hline & 17.656 & - & Routero & RIPv2 \\
\hline & 17.657 & Routero & Switcho & RIPv2 \\
\hline & 17.657 & Routero & Router1 & RIPv2 \\
\hline & 17.657 & Routero & Router2 & RIPv2 \\
\hline$\infty$ & 17.658 & Switcho & $\mathrm{PCO}$ & RIPv2 \\
\hline
\end{tabular}

Fig. 4 Convergence Time of RIP V2

\begin{tabular}{|c|c|c|c|c|}
\hline \multicolumn{5}{|c|}{ Simulation Panel } \\
\hline \multicolumn{5}{|c|}{ Event List } \\
\hline \multirow[t]{22}{*}{ Vis. } & Time(sec) & Last Device & At Device & Type \\
\hline & 2.821 & - & Router1 & OSPF \\
\hline & 2.822 & Router1 & Routero & OSPF \\
\hline & 4.196 & - & Routero & OSPF \\
\hline & 4.197 & Router0 & Router1 & OSPF \\
\hline & 5.635 & - & Routero & OSPF \\
\hline & 5.636 & Router0 & Router2 & OSPF \\
\hline & 6.745 & - & Router2 & OSPF \\
\hline & 6.746 & Router2 & Router1 & OSPF \\
\hline & 8.543 & - & Router1 & OSPF \\
\hline & 8.544 & Router1 & Router2 & OSPF \\
\hline & 8.545 & - & Router2 & OSPF \\
\hline & 8.546 & Router2 & Router0 & OSPF \\
\hline & 8.996 & - & Router2 & OSPF \\
\hline & 8.997 & Router2 & Switch2 & OSPF \\
\hline & 8.998 & - & Routero & OSPF \\
\hline & 8.998 & Switch2 & $\mathrm{PC2}$ & OSPF \\
\hline & 8.999 & Router0 & Switcho & OSPF \\
\hline & 9.000 & Switch0 & $\mathrm{PCO}$ & OSPF \\
\hline & 9.474 & - & Router1 & OSPF \\
\hline & 9.475 & Router1 & Switch1 & OSPF \\
\hline & 9.476 & Switch1 & PC1 & OSPF \\
\hline
\end{tabular}

Fig. 5 Convergence Time of OSPF Protocol

EIGRP protocol: EIGRP uses the metric structure, based on these components: Bandwidth, Delay, Reliability, Load and 


\section{International Journal of Engineering Applied Sciences and Technology, 2021 \\ Vol. 6, Issue 5, ISSN No. 2455-2143, Pages 115-120 \\ Published Online September 2021 in IJEAST (http://www.ijeast.com)}

Maximum transmission unit. By default, only bandwidth and delay are used in the metric computation and the other values are turned off. The metric value of EIGRP is calculated by using the formula:

\section{Metric $=(\mathrm{K} 1 *$ Bandwidth $+\mathrm{K} 2 *$ Delay $)$}

Convergence time of EIGRP configured network is shown below.

\begin{tabular}{|c|c|c|c|c|}
\hline \multicolumn{5}{|c|}{ Event List } \\
\hline Vis. & Time(sec) & Last Device & At Device & Type \\
\hline & 0.276 & - & Router2 & EIGRP \\
\hline & 0.277 & Router2 & Router0 & EIGRP \\
\hline & 1.574 & - & Router0 & EIGRP \\
\hline & 1.575 & Router0 & Router1 & EIGRP \\
\hline & 1.823 & - & Router0 & EIGRP \\
\hline & 1.824 & Routero & Router2 & EIGRP \\
\hline & 3.404 & - & Router2 & EIGRP \\
\hline & 3.405 & Router2 & Router1 & EIGRP \\
\hline & 3.599 & - & Router1 & EIGRP \\
\hline & 3.600 & Router1 & Router0 & EIGRP \\
\hline & 4.468 & - & Router1 & EIGRP \\
\hline & 4.469 & Router1 & Router2 & EIGRP \\
\hline & 4.724 & - & Router2 & EIGRP \\
\hline & 4.725 & Router2 & Router0 & EIGRP \\
\hline
\end{tabular}

Fig. 6 Convergence time of EIGRP

From the above observation, for the network topology configured, EIGRP takes $4.725 \mathrm{sec}$ to converge. Compared to RIP v2 convergence time OSPF convergence time EIGRP converges faster.

EIGRP supports a maximum of 255 routers in the network, which is better than RIP v2.EIGRP is used in medium and large scale networks. Administrative distance of EIGRP is 90 and that of RIP v2 and OSPF are 120 and 110 respectively. Administrative Distance counts the reliability of a routing protocol. A smaller Administrative Distance (AD) is more trusted by a router, therefore the best Administrative Distance (AD) being 0 and the worst, 255.

\section{B. IP traffic management using standard ACL}

Standard access control lists (ACLs) are router configuration scripts that control whether a router permits or denies packets based on the source address and thus controlling the traffic towards the web server. Standard ACL can filter only based on source address.

Consider Fig. 2 network topology where the following network policies are implemented on R2:

- $\quad$ The 192.168.11.0/24 network is not allowed access to the Webserver on the 192.168.20.0/24 network.
- All other access is permitted.

The following network policies are implemented on R3:

- The 192.168.10.0/24 network is not allowed to communicate to the $192.168 .30 .0 / 24$ network.

- $\quad$ All other access is permitted.

Only, ping from PC1 and PC3 to web server is permitted and ping from $\mathrm{PC} 2$ to web server is denied using standard ACL

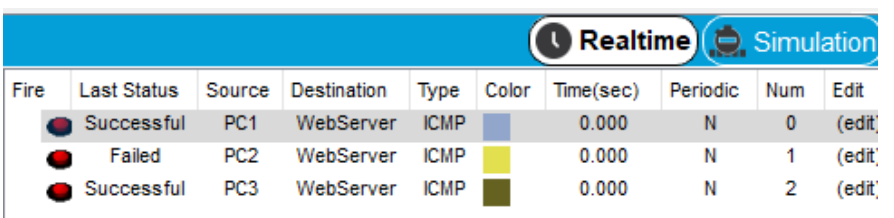

Fig. 7 Access and denial of packets from hosts using standard ACL

\section{IP traffic management using Extended ACL}

Extended Access Control Lists (ACLs) allow you to permit or deny traffic from specific IP addresses to a specific destination IP address and port. It also allows you to specify different types of traffic such as ICMP, TCP, UDP, etc. Needless to say, it is very granular and allows you to be very specific.

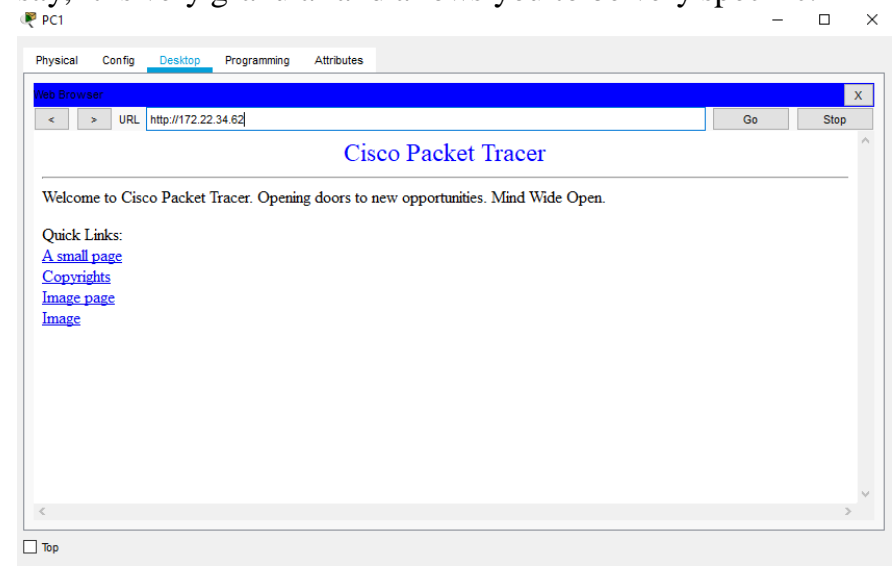

Fig. 8 Allowing access for http application

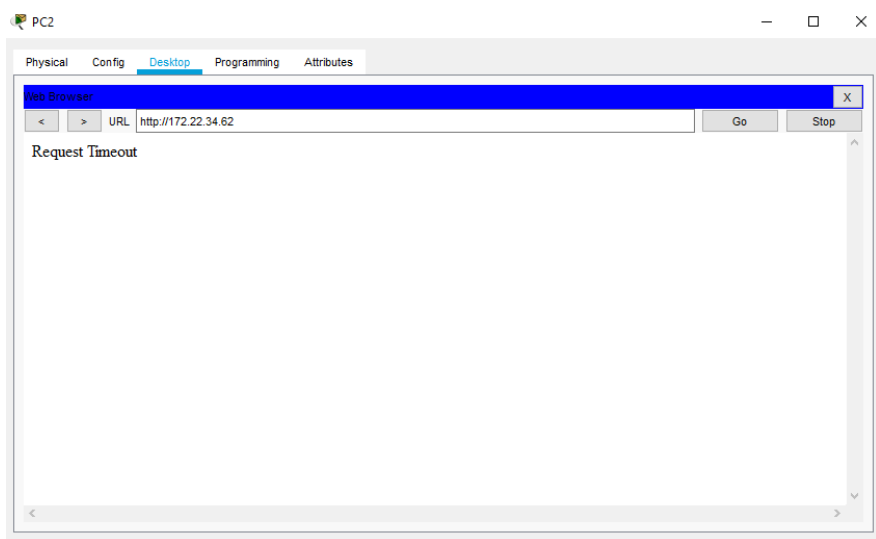

Fig. 9 Denying access for http application 


\section{REFERENCES}

[1] C. G. Dumitrache ; G. Predusca ; L. D. Circiumarescu ; N. Angelescu ; D. C. Puchianu 'Comparative study of RIP, OSPF and EIGRP protocols using Cisco Packet Tracer', 5th International Symposium on Electrical and Electronics Engineering (ISEEE),2017.

[2] Nurhayati ; Rahmat Fajar Al Farizky "Routing protocol RIPng, OSPFv3, and EIGRP on IPv6 for video streaming services" 5th International Conference on Cyber and IT Service Management (CITSM) ,2017.

[3] Kavya Altefi; Amir Hossein Shahin ; Saadiah Yahya ; Alireza Erfanian "Performance evaluation of RIP and EIGRP Routing Protocols in IEEE 802.3u standard" 3rd International Conference on Computer and Information Sciences (ICCOINS), 2016.

[4] Shipra Suman, Er. Aditi Agrawal "IP Traffic Management with Access Control List Using Cisco Packet Tracer", International Journal of Science, Engineering and Technology Research (IJSETR) Volume 5, Issue 5, May 2016.

[5] Lalu Zazuli Azhar Mardedi Abidarin Rosidi "Developing Computer Network Based on EIGRP Performance Comparison and OSPF" (IJACSA) International Journal of Advanced Computer Science and Applications, Vol. 6, No. 9, 2015.

[6] Michael, N.; Ao Tang; DahaiXu,C C chiang presents "Optimal link-state hop-by-hop routing," Network Protocols (ICNP), 2013 21st IEEE International Conference on , vol., no., pp.1,10, 7-10 Oct. 2013. 